\title{
A saúde mental dos profissionais de saúde frente à pandemia do COVID-19: uma revisão integrativa
}

\author{
The mental health of health professionals in front of the COVID-19 pandemic: an \\ integrative review
}
La salud mental de los profesionales de la salud delante de la pandemia COVID-19: una revisión integradora

Amanda Dornelas Prado ${ }^{1 *}$, Bruna Cristina Peixoto ${ }^{1}$, Andréa Mara Bernardes da Silva ${ }^{1}$, Luana Araújo Macedo Scalia ${ }^{1}$.

\section{RESUMO}

Objetivo: Conhecer a situação da saúde mental dos profissionais da área da saúde da linha de frente na pandemia do COVID-19, e quais consequências para os serviços de saúde. Métodos: Trata-se de uma revisão integrativa de literatura e foi realizada nas bases de dados LILACS, MEDLINE pela biblioteca virtual da Saúde, na PUBMED e Scielo, usando descritores: Saúde mental / Mental health / Salud mental, Covid - 19 / Covid - 19 / Covid - 19, Profissional de saúde/ Health Personnel / Personal de Salud, Serviços de Saúde / Health Services / Servicios de Salud. Resultados: Foram selecionados 8 artigos para essa revisão. A partir da leitura dos artigos percebeu-se a fragilidade na saúde mental do trabalhador da saúde, dentro de incertezas do futuro e da cura do vírus COVID-19. Dessa forma, percebe-se altos índices de ansiedade, estresse, depressão, medo, angústia e sono alterado, doenças e muitas vezes esse profissional não procura ajuda para si mesmo. Considerações finais: $O$ índice de sintomas psiquiátricos que já é alarmante normalmente no trabalhador da saúde, durante a pandemia tem se agravado. As organizações de Saúde precisam se atentar a isso e organizar maneiras de melhorar o bem-estar desses profissionais.

Palavras-chave: Infecções por coronavirus, Profissionais da saúde, Saúde mental, Serviços de saúde.

\section{ABSTRACT}

Objective: To analyze and understand the mental health situation of frontline health professionals in this COVID-19 pandemic. Methods: This is a literature review, the research was conducted in the virtual library of the VHL, using the databases, LILACS, MEDLINE, PUBMED and Scielo. Using controlled descriptors: Saúde mental / Mental health / Salud mental, Covid - 19 / Covid - 19 / Covid - 19, Profissional de saúde/ Health Personnel / Personal de Salud, Serviços de Saúde / Health Services / Servicios de Salud. At the conclusion of the searches, 8 articles were selected for this review. Results: From the reading of the articles, the fragility of the professional's mental health was perceived, within the uncertainties of the future and the cure, mental illnesses get worse, and many times this professional does not seek help for himself. Conclusion: This study provided the opportunity to investigate and analyze how the mental health of those who are facing COVID-19 de is displayed, and I could see that these professionals need greater attention.

Key words: Coronavirus infections, Health professionals, Mental health, Health services.

\section{RESUMEN}

Objetivo: Analizar y comprender la situación de salud mental de los profesionales de salud de primera línea en esta pandemia de COVID-19. Métodos: Esta es una revisión de la literatura, la investigación se realizó en la biblioteca virtual de la BVS, utilizando las bases de datos, LILACS, MEDLINE, PUBMED y Scielo. Uso de descriptores controlados: Saúde mental / Mental health / Salud mental, COVID-19 / COVID-19 / COVID-19, Profissional de saúde / Health Personnel / Personal de Salud, Serviços de Saúde / Health Services / Servicios de Salud. Boleanos Al final de las búsquedas, se seleccionaron 8 artículos para esta revisión. Resultado: a partir de la lectura de los artículos, se percibió la fragilidad de la salud mental del profesional, dentro de las incertidumbres del futuro y la cura, las enfermedades mentales empeoran, y muchas veces este profesional na busca ayuda para sí mismo. Conclusión: Este estudio brindó la oportunidad de investigar y analizar cómo se muestra la salud mental de quienes se enfrentan a COVID-19 de y pude ver que estos profesionales necesitan mayor atención.

Palabras clave: Infecciones por coronavirus, Profesionales de la salud, Salud mental, Servicios de salud.

1Universidade Federal de Uberlândia (UFU), Uberlândia - MG. *E-mail: amandauberlandia@hotmail.com 


\section{INTRODUÇÃO}

Em dezembro de 2019, a Organização Mundial da Saúde (OMS) foi alertada de um novo vírus que desencadeava vários casos de pneumonia na cidade de Wuhan, província de Hubei, na República Popular da China. Esse novo vírus ainda não tinha sido identificado em humanos. Uma semana depois, em janeiro de 2020 o COVID-19 já era a segunda principal causa de resfriado comum (após rinovírus) (OPAS, 2020).

De acordo com o Protocolo de Manejo Clínico do Coronavírus, o vírus foi denominado coronavírus 2 da síndrome respiratória aguda grave (SARS-CoV-2) e produz a doença classificada como COVID-19 (LI Q, et al., 2020). Os sintomas com maior frequência são febre, tosse seca, cansaço, coriza, obstrução nasal, dor de garganta e diarreia, sendo que $14 \%$ dos pacientes apresentam sintomas severos (dificuldade em respirar e falta de ar), necessitando de internação para oxigenoterapia e 5\% apresentam sintomas críticos (insuficiência respiratória, risco de morte, entre outros) (SBI, 2020).

Em 30 de janeiro de 2020, a OMS declarou que o surto do COVID-19 constituía uma Emergência de Saúde Pública de importância internacional, o mais alto nível de alerta, conforme previsto no Regulamento Sanitário Internacional (OMS, 2020). De acordo com o mapa interativo do Centro de Ciência e Engenharia de Sistemas (CSSE) na Universidade Johns Hopkins, já são 6.399.230 casos conformados e 384.463 mortes em 188 países (DONG E, et al., 2020).

Na América Latina o COVID-19 chegou mais tarde que em outros continentes. No Brasil o primeiro caso registrado foi em 25 de fevereiro de 2020, porém atualmente é o país com maior número de casos e de mortes do continente (DONG E, et al., 2020), e provavelmente esses dados são subestimados pois o país não tem testes suficientes.

Barroso AlL, et al. (2020) fizeram um mapeamento mostrando o índice de risco que os trabalhadores brasileiros têm de serem contaminados pelo COVID-19 durante suas atividades profissionais e os trabalhadores da saúde apresentaram de 97 a 100\% de risco de contágio desde técnicos de saúde bucal a técnicos de enfermagem, enfermeiros e médicos.

De acordo com o Ministério da Saúde (2020), no Boletim Epidemiológico Especial no 16 divulgado em maio no Brasil, existem 199.768 profissionais de saúde registrados no e-SUS Notifica com suspeita de COVID-19 sendo que $31.790(15,9 \%)$ foram confirmados para a doença, $53.677(26,9 \%)$ foram descartados por não atenderem aos critérios clínico-laboratoriais e 114.301 (57,2\%) permaneceram em investigação.

A categoria profissional com o maior número de registros no sistema foi "Técnico ou Auxiliar em Enfermagem" (68.250 ou 34,2\%), seguida de "Enfermeiro" (33.733 ou 16,9\%), "Médico" (26.546 ou 13,3\%), "Recepcionista" (8.610 ou 4,3\%) e "Outro tipo de agente de saúde" (5.013 ou 2,5\%) (BRASIL, 2020).

Os profissionais de saúde lidam a todo o tempo com a morte e com decisões difíceis que podem afetar seu bem estar físico e mental. Segundo a OMS, "A saúde mental é definida como um estado de bem-estar no qual cada indivíduo realiza seu próprio potencial pode lidar com o estresse normal da vida, pode trabalhar de maneira produtiva e é capaz de contribuir com sua comunidade" (WHO, 2014).

Devido a esse rápido crescimento do número de profissionais de saúde infectados pelo COVID-19 e todo o estresse e pressão que têm sofrido, a saúde mental desses profissionais tem sido apontada como uma grande preocupação.

Em estudo realizado no Canadá no surto de COVID-19, Pereira MD, et al. (2020) encontraram sintomas que exemplificam prejuízo na saúde mental dos trabalhadores da saúde, como sensação de alto risco de contaminação, efeito da doença na vida profissional e humor deprimido.

Além disso a exaustão física e mental, a dor da perda de pacientes e colegas, a dificuldade de tomada de decisão, o medo da contaminação e da transmissão da doença aos entes próximos também são fatores que prejudicam a saúde mental dos profissionais atuantes na linha de frente da doença (GUIMARÃES AV e BRASIL AM, 2018).

Diante disso, o objetivo dessa pesquisa foi entender, através de uma revisão integrativa, como o COVID19 afeta a saúde mental dos profissionais da área da saúde e quais os impactos no serviço de saúde. 


\section{MÉTODOS}

Este estudo é uma revisão de literatura baseada em artigos publicados na literatura até abril de 2020. A pergunta da pesquisa foi elaborada através estratégia PICO (SANTOS CMC, et al., 2007). Segundo Santos et al. (2007), a PICO representa um acrônimo para Paciente, Intervenção, Comparação e "Outcomes" (desfecho), sendo esta: "Como o COVID-19 afeta a saúde mental dos profissionais da área da saúde no mundo e quais os impactos no serviço de saúde?".

A busca de artigos foi realizada no banco de dados da Biblioteca Virtual em Saúde (BVS), na base de dados Latino-Americana e do Caribe em Ciências da Saúde (LILACS), Sistema Online de Busca e Análise de Literatura Médica (MEDLINE) e nas bases de dados PUBMED e Scielo, utilizando os seguintes descritores controlados: Saúde mental / Mental health / Salud mental, Covid - 19 / Covid - 19 / Covid - 19, Profissional de saúde/ Health Personnel / Personal de Salud, Serviços de Saúde / Health Services / Servicios de Salud. Boleanos.

Como critério de inclusão foram selecionados artigos originais, nos idiomas em português, inglês e espanhol, no espaço temporal de 2019 a 2020 e que respondiam à questão norteadora. Foram excluídos artigos duplicados, artigos de revisão de literatura, relato de caso, artigos que antecediam o tempo estipulado para inclusão.

\section{RESULTADOS}

Após a busca nas bases de dados e excluídos artigos que não era de 2019 e 2020, foram selecionados 523 artigos. Após a leitura dos títulos foram separados 131 artigos. Após a leitura do resumo, foram selecionados 25 estudos. Esses 25 foram lidos na íntegra e, oito artigos foram incluídos para a discussão acerca do tema, como mostrado fluxograma a seguir (Figura 1).

Figura 1 - Fluxograma da seleção dos artigos da revisão integrativa, a partir dos critérios de inclusão e exclusão.

COVID-19 AND saúde mental AND profissionais da saúde (20 artigos)

COVID-19 AND saúde mental AND serviços de saúde (45 artigos)

COVID-19 AND saúde mental (173 artigos)

COVID-19 AND profissionais da saúde (72 artigos)

COVID-19 AND serviços de saúde (213 artigos)

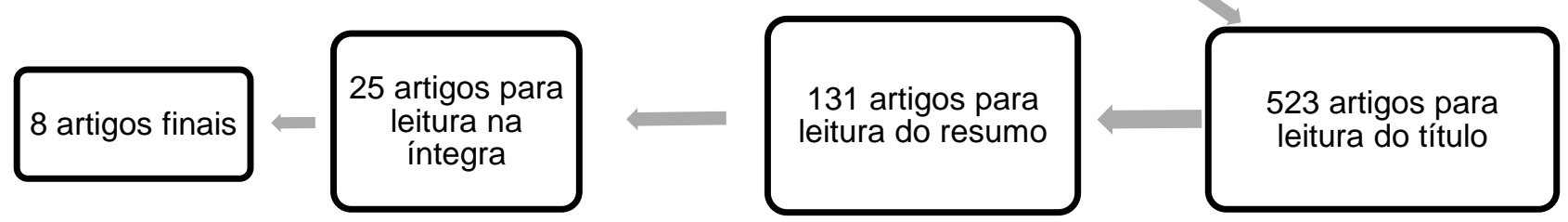

Fonte: Prado AD, et al., (2020).

Dentre os oito selecionados e incluídos na Revisão Integrativa a partir da leitura na íntegra, todos foram publicados em 2020, todos os artigos no idioma Língua Inglesa e de periódicos internacionais. Três estudos (37,5\%) foram publicados na revista Psychiatry Research. Cinco estudos $(62,5 \%)$ foram realizados na China, e os outros três na Austrália, Malásia e Itália (Quadro 1).

Baseado na pergunta norteadora, dividiu-se a discussão em 3 categorias temáticas, sendo: 1) Saúde mental dos profissionais da saúde; 2) Impactos da pandemia aos serviços de saúde e 3) Formas de enfrentamento dos profissionais de saúde. 


\section{Revista Eletrônica Acervo Saúde / Electronic Journal Collection Health ｜ ISSN 2178-2091}

Quadro 1 - Apresentação dos estudos incluídos na revisão integrativa, segundo o título, autores, ano da publicação, nacionalidade, metodologia e nível de evidência.

\begin{tabular}{|c|c|c|c|c|c|c|}
\hline Título & Autores & Ano & País & Periódico & Método & Nível de Evidência \\
\hline Lições aprendidas com a pandemia COVID-19 & KHOO EJ & 2020 & Malásia & Acta Pædiatrica & Artigo de Opinião & V \\
\hline $\begin{array}{l}\text { Serviços de Saúde Mental na Lombardia durante o } \\
\text { surto de COVID-19 }\end{array}$ & $\begin{array}{l}\text { PERCUDANI M, et } \\
\text { al. }\end{array}$ & 2020 & Itália & $\begin{array}{l}\text { Psychiatry } \\
\text { Research }\end{array}$ & $\begin{array}{l}\text { Relato de } \\
\text { experiência }\end{array}$ & V \\
\hline $\begin{array}{l}\text { Impacto na saúde mental e percepções de cuidados } \\
\text { psicológicos entre médicos e enfermeiros em Wuhan } \\
\text { durante o novo surto de doença coronavírus 2019: } \\
\text { Estudo transversal }\end{array}$ & KANG L, et al. & 2020 & China & $\begin{array}{l}\text { Brain, behavior, } \\
\text { and immunity }\end{array}$ & $\begin{array}{l}\text { Estudo } \\
\text { transversal }\end{array}$ & IV \\
\hline $\begin{array}{l}\text { Consultas remotas na era da pandemia COVID-19: } \\
\text { Experiência preliminar em um ambiente regional } \\
\text { australiano de atenção à saúde mental aguda }\end{array}$ & $\begin{array}{l}\text { KAVOOR AR; } \\
\text { CHAKRAVARTHY } \\
\text { K; JOHN T }\end{array}$ & 2020 & Austrália & $\begin{array}{l}\text { Asian Journal of } \\
\text { Psychiatry }\end{array}$ & $\begin{array}{l}\text { Relato de } \\
\text { experiência }\end{array}$ & V \\
\hline $\begin{array}{c}\text { Transtorno de ansiedade generalizada, sintomas } \\
\text { depressivos e qualidade do sono durante o surto de } \\
\text { COVID-19 na China: uma pesquisa transversal } \\
\text { baseada na web }\end{array}$ & $\begin{array}{l}\text { HUANG Y; ZHAO } \\
\text { N }\end{array}$ & 2020 & China & $\begin{array}{l}\text { Psychiatry } \\
\text { Research }\end{array}$ & $\begin{array}{l}\text { Estudo } \\
\text { transversal }\end{array}$ & IV \\
\hline $\begin{array}{l}\text { Fatores associados aos desfechos de saúde mental } \\
\text { entre profissionais de saúde expostos à doença } \\
\text { coronavírus } 2019\end{array}$ & LAl J, et al. & 2020 & China & JAMANetworkOpen & $\begin{array}{l}\text { Estudo } \\
\text { transversal }\end{array}$ & IV \\
\hline $\begin{array}{c}\text { Estado psicológico da força de trabalho médica } \\
\text { durante a pandemia COVID-19: Estudo transversal }\end{array}$ & LU W, et al. & 2020 & China & $\begin{array}{l}\text { Psychiatry } \\
\text { Research }\end{array}$ & $\begin{array}{l}\text { Estudo } \\
\text { transversal }\end{array}$ & IV \\
\hline $\begin{array}{l}\text { Sintomas psicológicos entre profissionais de saúde } \\
\text { da linha de frente durante surto de COVID-19 em } \\
\text { Wuhan }\end{array}$ & DU J, et al. & 2020 & China & $\begin{array}{l}\text { General Hospital } \\
\text { Psychiatry }\end{array}$ & $\begin{array}{l}\text { Estudo } \\
\text { transversal }\end{array}$ & IV \\
\hline
\end{tabular}

Fonte: Prado AD, et al., 2020. 
Quadro 2 - Apresentação dos estudos incluídos na revisão integrativa, segundo o tamanho da amostra, instrumentos utilizados e principais achados.

\begin{tabular}{|c|c|c|c|}
\hline Autores & Tamanho da amostra & Instrumentos Utilizados & Principais achados \\
\hline $\begin{array}{l}\text { KANG } \\
L \text {, et al. }\end{array}$ & $\begin{array}{l}994 \text { participantes sendo } \\
183(18,4 \%) \text { médicos e } \\
811(81,6 \%) \\
\text { enfermeiras }\end{array}$ & $\begin{array}{l}\text { Dados demográficos, Questionário de Saúde do } \\
\text { Paciente de } 9 \text { itens, Transtorno de Ansiedade } \\
\text { Generalizada, Índice de Gravidade da Insônia de } \\
7 \text { itens e Escala de Impacto do Evento de } 22 \text { itens }\end{array}$ & $\begin{array}{c}\text { Trinta e seis por cento apresentava distúrbios de saúde mental abaixo } \\
\text { do limiar, } 34,4 \% \text { tinham distúrbios leves, } 22,4 \% \text { tinham distúrbios } \\
\text { moderados e } 6,2 \% \text { apresentavam distúrbios graves. }\end{array}$ \\
\hline $\begin{array}{l}\text { HUANG } \\
\text { Y; } \\
\text { ZHAO N }\end{array}$ & $\begin{array}{l}\text { Dos } 7.236 \text { participantes, } \\
2.250(31,1 \%) \text { dos } \\
\text { participantes eram } \\
\text { profissionais de saúde }\end{array}$ & $\begin{array}{c}\text { Questionário demográfico, conhecimento } \\
\text { relacionado ao COVID-19, versão chinesa da } \\
\text { escala Transtorno de Ansiedade Generalizada- } \\
\text { 7, Escala Epidemiológica para Depressão e a } \\
\text { versão chinesa do Índice de Qualidade do Sono } \\
\text { de Pittsburgh }\end{array}$ & $\begin{array}{l}\text { A prevalência global de ansiedade, sintomas depressivos e qualidade } \\
\text { do sono foram de } 35,1 \%, 20,1 \% \text { e } 18,2 \% \text {, respectivamente. Em } \\
\text { comparação com outros grupos ocupacionais, os profissionais de } \\
\text { saúde }(23,6 \%) \text { relataram a maior taxa de má qualidade do sono. }\end{array}$ \\
\hline $\begin{array}{l}\text { LAI J, et } \\
\text { al. }\end{array}$ & $\begin{array}{l}1257 \text { profissionais de } \\
\text { saúde em } 34 \text { hospitais }\end{array}$ & $\begin{array}{l}\text { Versões chinesas do Questionário de Saúde do } \\
\text { Paciente de } 9 \text { itens, escala de Transtorno de } \\
\text { Ansiedade Generalizada de } 7 \text { itens, o Índice de } \\
\text { Gravidade da Insônia de } 7 \text { itens e o Impacto da } \\
\text { Escala de Eventos de } 22 \text { itens }\end{array}$ & $\begin{array}{c}60,8 \% \text { eram enfermeiros, } 39,2 \% \text { eram médicos; } 60,5 \% \text { trabalhavam } \\
\text { em hospitais em Wuhan, e } 41,5 \% \text { eram profissionais de saúde de linha } \\
\text { de frente. } 50,4 \% \text { relataram sintomas de depressão, } 44,6 \% \text { de } \\
\text { ansiedade, } 34 \% \text { de insônia e } 71,5 \% \text { de angústia. Enfermeiras, } \\
\text { mulheres, profissionais de saúde de linha de frente e aqueles que } \\
\text { trabalham em Wuhan, relataram graus mais graves de todas as } \\
\text { medidas de sintomas de saúde mental }\end{array}$ \\
\hline $\begin{array}{l}\text { LU W, } \\
\text { et al. }\end{array}$ & $\begin{array}{l}2299 \text { participantes } \\
\text { sendo } 2042 \text { equipe } \\
\text { médica (médicos e } \\
\text { enfermeiros) e } 257 \\
\quad \text { funcionários } \\
\quad \text { administrativos } \\
\text { (incluindo a logística) }\end{array}$ & $\begin{array}{l}\text { A escala numérica de classificação por } \\
\text { medo, Escala de Ansiedade de Hamilton e } \\
\text { Escala de Depressão de Hamilton }\end{array}$ & $\begin{array}{l}\text { A equipe médica com medo moderado e grave foi maior do que no } \\
\text { grupo de pessoal administrativo ( } 70,6 \% \text { VS } 58,4 \% \text { ). Além disso, } 22,6 \% \\
\text { da equipe médica apresentou ansiedade leve a moderada e } 2,9 \% \\
\text { foram graves, as proporções de resposta dos funcionários } \\
\text { administrativos foram de } 17,1 \% \text { e } 2,9 \% \text {. As diferenças foram } \\
\text { significativas. Além disso, } 11,8 \% \text { da equipe médica apresentava } \\
\text { depressão leve a moderada, e } 0,3 \% \text { com depressão grave. }\end{array}$ \\
\hline $\begin{array}{l}\text { DU J, et } \\
\text { al. }\end{array}$ & $\begin{array}{l}\text { Os participantes foram } \\
\text { trabalhadores da saúde } \\
\text { de linha de frente de } \\
\text { dois hospitais baseados } \\
\text { em Wuhan }(n=60) \text { e de } \\
\text { dois hospitais externos } \\
\quad(n=74)(n=134)\end{array}$ & $\begin{array}{l}\text { Dados demográficas, qualidade do sono, estresse } \\
\text { pela Escala de Estresse Percebido, Depressão } \\
\text { pelo Inventário de Depressão Beck-II }\end{array}$ & $\begin{array}{c}\text { Prevalência de depressão elevada e sintomas de ansiedade: } 12,7 \% \text { e } \\
20,1 \% \text {, respectivamente. Mais da metade }(59,0 \%) \text { apresentaram níveis } \\
\text { moderados a severos de estresse percebido. Os sintomas depressivos } \\
\text { e de ansiedade foram mais comuns entre as mulheres, aqueles que } \\
\text { estavam menos preparados psicologicamente, falta de auto eficácia } \\
\text { percebida e sem apoio familiar, bem como aqueles com baixa } \\
\text { qualidade do sono. }\end{array}$ \\
\hline
\end{tabular}

Fonte: Prado AD, et al., 2020.

REAS/EJCH | Vol.Esp.46 | e4128 | DOI: https://doi.org/10.25248/reas.e4128.2020 Página 5 de 9




\section{DISCUSSÃO}

\section{Saúde mental dos profissionais da saúde}

O surto epidemiológico do vírus nomeado de Síndrome Respiratória Aguda Grave Coronavírus 2 (SARSCoV-2) iniciou-se sobre a cidade de Wuhan, capital da província de Hubei, localizada na China em dezembro de 2019 (CHAN JF-W, et al., 2020). Isso explica o fato de encontrar a maioria dos artigos publicados neste país, pois foram os primeiros também a encontrar evidências sobre o assunto. Em Janeiro, o governo Chinês declarou a existência da doença e sua rápida disseminação na população, tomando medidas preventivas rígidas para que evitassem riscos maiores como os casos de pneumonia em pessoas testadas positivas para o COVID-19, com alto índice de óbito (CHEN Q, et al., 2020a; PHELAN AL, et al., 2020; ZHANG et al., 2020b).

Na sequência, no mês de fevereiro de 2020, o setor de saúde decretou estado de calamidade pandêmica quando a COVID-19 chegou à região de Lombardia, no norte da Itália, tornando-se rapidamente a região mais afetada ao nível mundial. Logo se espalhou e hoje já são 188 países afetados (REMUZZI A e REMUZZI G, 2020; EPICENTRO, 2020).

Diante desse cenário da pandemia e alta disseminação e mortalidade, os profissionais da saúde que ficaram diretamente ligados aos pacientes infectados e envolvidos tanto no diagnóstico, tratamento e no atendimento em geral, mostrou-se com altos índices de sofrimento psíquico como medo, ansiedade, depressão, angústia, sono prejudicado e outros sentimentos relacionados ao risco à exposição do vírus. Isso foi demonstrado nos cinco artigos encontrados nesta pesquisa, sobre saúde mental dos trabalhadores de saúde na China (Quadro 2).

Nesses cinco trabalhos encontrou-se índices de estresse moderado a grave em $59 \%$ dos trabalhadores de saúde, depressão em $12,7 \%$ a $50,4 \%$, e ansiedade de $20,1 \%$ a $44,6 \%$ desses profissionais. Além disso, os sentimentos de angústia e medo e sono prejudicado é também maior nessa população de estudo.

Dentre os estudos populacionais já realizados até o presente momento sobre implicações na saúde mental diante da pandemia do novo coronavírus, destaca-se o de Huang Y e Zhao N (2020) que avaliaram a saúde mental da população chinesa após o surto do COVID-19 através de pesquisa online. Participaram da pesquisa 7236, sendo que $2.250(31,1 \%)$ eram profissionais de saúde. A prevalência geral de ansiedade, sintomas depressivos e qualidade do sono foram de $35,1 \%, 20,1 \%$ e $18,2 \%$, respectivamente sendo que em comparação com outros grupos ocupacionais, os profissionais de saúde $(23,6 \%)$ relataram a maior taxa de má qualidade do sono (HUANG Y e ZHAO N, 2020).

Da mesma forma, Lai J, et al. (2020) relataram que 34\% dos médicos e enfermeiras apresentaram insônia e $71,5 \%$ angústia. Enfermeiras, mulheres, profissionais de saúde de linha de frente relataram graus mais graves de todas as medidas de sintomas de saúde mental.

Du J, et al. (2020) também encontraram que os sintomas depressivos e de ansiedade foram mais comuns entre as mulheres, aqueles que estavam menos preparados psicologicamente, falta de auto eficácia percebida e sem apoio familiar, bem como aqueles com baixa qualidade do sono. De fato, é muito importante conhecer a população mais vulnerável para que se possa agir com mais intensidade e de forma mais efetiva, buscando formas de auxiliar na diminuição dos impactos da pandemia.

Uma análise interessante realizada por Kang L, et al. (2020) foi que esses índices de sintomas de depressão e ansiedade variava dependendo da quantidade de exposição à pessoas infectadas. Assim, 0 grupo com escores para doença mental abaixo do limite teve contato com menos pessoas confirmadas ou suspeitas de estarem infectadas pelo vírus. O grupo com um nível mais alto de angústia teve um escopo de exposição mais extenso. Também houve diferenças significativas ao acesso dos serviços de saúde mental entre os grupos, sendo que aqueles com distúrbios graves acessaram menos materiais e recursos psicológicos disponíveis na mídia (KANG L, et al., 2020).

Trabalhadores da saúde de outro estudo também relataram que suas maiores preocupações e que afetavam a saúde mental era a alta taxa de transmissão do vírus entre a população e seu alto risco de mortalidade (LAI J, et al., 2020). Interessante que os autores pesquisaram profissionais da saúde de hospitais 
com altas taxas de COVID-19 e outros com baixas ou sem casos do vírus, demonstrando que os da linha de frente na pandemia apresentavam mais sintomas de doenças mentais.

Da mesma forma, Lu W, et al (2020) comparando os valores médios de medo, os de ansiedade e depressão entre dois grupos, identificou que a equipe médica revelou maior medo, ansiedade e depressão do que a equipe administrativa. A análise adicional mostrou que a equipe médica que trabalha nos departamentos: respiratório, pronto-socorro, unidade de terapia intensiva e departamento de doenças infecciosas, que entram em contato direto com pacientes com pneumonia por coronavírus, revelou mais distúrbios psicológicos e tinha quase duas vezes mais o risco de sofrer ansiedade e depressão, em comparação com a equipe não clínica, com pouca possibilidade de contato com pacientes com pneumonia por coronavirus (LU W, et al., 2020).

Porém, não é apenas o risco de infecção e desconhecimento do vírus que tem causado esse estresse. A maioria destes profissionais estão em longas jornadas de trabalho, execução de vários plantões consecutivos, falta de Equipamentos de Proteção Individual (EPI) para sua própria proteção, ampla cobertura da imprensa, baixo estoque de medicamentos e falta de apoio por todos envolvidos na situação pandêmica (KANG $L$, et al., 2020).

Além do receio do próprio contágio, esses profissionais da saúde temiam a infecção à sua família, colegas de trabalho e demais amigos, sentindo incertezas e rotulações, relutâncias em ir trabalhar e altos índices de pedidos de demissão. Houve relatos de profissionais que diziam que sentiram emoções nunca vivenciadas. Esses profissionais estão trabalhando isoladamente, em alta exposição aos riscos e tem e/ou tiveram contato com colegas de trabalho que estão/foram contaminados e alguns foram ao óbito, em um período de aproximadamente quatro meses (KANG L, et al., 2020).

Huang Y e Zhao N (2020) ainda relataram que a maioria dos trabalhadores da saúde na China voltou a trabalhar para lidar com o desenvolvimento da doença e que uma razão possível para o adoecimento é que o tempo de trabalho e a intensidade do trabalho dos profissionais de saúde aumentaram, fazendo com que não tenham tempo suficiente para descansar e sejam propensos a estresse crônico e sofrimento psicológico. Em casos graves, pode ocorrer um sintoma de transtorno de estresse pós-traumático (TEPT), que está altamente correlacionado com falta de sono (HUANG Y e ZHAO N, 2020).

Diante disso, não há como deixar de citar o sofrimento moral. O sofrimento de ter que talvez escolher entre quem vive e quem morre. Conflitos e sentimentos contraditórios para os profissionais de saúde e para o público em geral. O sofrimento moral afeta todos nós e deve ser respeitado e discutido abertamente. Esse sofrimento moral é um sinal saudável, não patológico. Reconhecimento significa que estamos tentando fazer a coisa certa, sabemos que às vezes não podemos, mas devemos continuar (KHOO EJ, 2020).

Nas últimas semanas, se tem tomado cautelas à curto e longo prazo com a saúde mental dos profissionais de saúde que estão diretamente ligados à população contaminada com o COVID-19. Tem-se pensado em troca de setor de trabalho ou afastamento de trabalhadores alto risco, como aqueles com problemas cardíacos e respiratórios, diabéticos, idade acima de 60 anos e etc., além da redução do horário diário de trabalho e com maiores períodos de descanso, maior contratação e antecipação de formandos nas Ciências da Saúde nas Faculdades, monitoramento bastante rigoroso para o controle de contaminação pelo vírus, fornecimento de equipamentos completos com alta segurança e orientação em como lidar com a rotina nos hospitais em pandemias (KHALID I, et al., 2016).

\section{Impactos da pandemia aos serviços de Saúde}

Sistemas de saúde em todo o mundo estão ficando sobrecarregados. Ao final de abril, já havia ultrapassado 4 milhões de casos confirmados mundialmente, tornando os setores de saúde cheios, com profissionais esgotados e em muitos locais sem revezamento de turnos devido à alta demanda e poucos médicos, enfermeiros, técnicos de enfermagem, fisioterapeutas e outros profissionais, para a manutenção dos serviços essenciais de saúde, como o de prestação de primeiros socorros, reanimação, intubação e demais processos necessários que chegassem aos hospitais trazidos por parentes ou via ambulância (KAVOOR AR, et al., 2020). 
Dois dos artigos encontrados falaram sobre como para os sistemas de saúde se adaptarem com a chegada da pandemia. São alterações em protocolos de atendimento e de fluxo de pacientes, toda uma adaptação que foi necessária. De acordo com Kavoor AR, et al. (2020) sempre que possível, as consultas foram realizadas usando telemedicina e as avaliações presenciais foram limitadas a pacientes de alto risco clínico ou àqueles que solicitaram isso após as discussões sobre a estratégia de controle de infecção dos serviços. Se surgia alguma emergência durante a sessão telefônica, os arranjos de emergência estavam em vigor, como entrar em contato com parentes ou ambulâncias. Um questionário de triagem para COVID-19 foi administrado no momento do encaminhamento ou triagem inicial.

Da mesma forma, Precudani M, et al. (2020) relataram a adaptação a serviços públicos de Saúde Mental na Lombardia, Itália. Foram implementadas diretrizes de segurança para a equipe médica e os pacientes, incluindo intervenções psicossociais remotas e telemedicina. As internações hospitalares por distúrbios psiquiátricos agudos em pacientes positivos para o COVID-19 precisaram de uma área dedicada na enfermaria psiquiátrica ou, alternativamente, uma enfermaria médica apoiada pela equipe psiquiátrica (PRECUDANI M, 2020).

A cada visita, as indicações para limitar a propagação da infecção são explicadas e ilustradas. As atividades que envolvem os familiares dos pacientes são reduzidas apenas àquelas consideradas essenciais e foram substituídas por comunicação virtual ou por áudio. O monitoramento de sintomas respiratórios e outros sintomas do COVID-19, incluindo temperatura, eram realizados diariamente. A equipe foi equipada $e$ treinada no uso de equipamentos de proteção individual para atendimento direto a pacientes com COVID-19 e casos suspeitos, com febre ou outros sintomas da infecção. Também foi criada uma sala de para vestir e despir (PERCUDANI M, 2020).

\section{Formas de Enfrentamento dos profissionais da Saúde}

Após as evidências científicas demostrarem que a equipe de saúde tem passado por sofrimento psicológico com a pandemia do COVID-19, percebeu-se a importância de tratamentos psicológicos ou psiquiátricos à essa população, uma vez que, o cuidado em saúde mental favorece a atuação do profissional no seu local de trabalho, e a ausência disso reduzirá o seu potencial de cuidado, aumentarão as chances de afastamentos, disseminações, mortes e consequências posteriores a crise desta pandemia (LAl J, et al, 2020; LU W, et al., 2020).

Kang L, et al (2020) demonstraram que do todos os trabalhadores de saúde, 36,3\% receberam materiais psicológicos, 50,4\% obtiveram ajuda psicológica por meio de mídia e 17,5\% participaram de aconselhamento psicológico em grupo. A equipe de médicos e enfermagem que tinham baixos escores de ansiedade e depressão preferia obter habilidades para ajudar a aliviar o sofrimento psíquico dos outros, enquanto os com ansiedade e depressão moderada a grave queriam obter habilidades para autoajuda e demonstravam desejos mais urgentes de buscar ajuda de psicoterapeutas e psiquiatras (KANG L, et al., 2020).

Esse resultado corrobora com a discussão de Khoo EJ (2020) que mostra a estigmatização generalizada sobre procurar ajuda psicológica e que tem impacto adverso na saúde pública. Ser um modelo de trabalhador no setor de saúde inclui se ajudar quando necessário e incentivar as pessoas a buscarem tratamento sem medo.

Para cada hospital seria importante ter equipes de saúde mental, incluindo psiquiatras, psicólogos, enfermeiros e outros profissionais de saúde para dar suporte no enfrentamento da tensão e reduzir o risco de ansiedade e depressão da equipe médica. Realizar organização abrangente de consultas psicológicas regular e por um longo tempo, para tratar aqueles profissionais que sofrerem de estresse pós-traumático (TEPT) (LU W, et al., 2020; PERCUDANI M, 2020).

De acordo com a OPAS (2020) a intervenção neste momento de crise deve ser baseada no acolhimento do sujeito e de suas emoções, de forma sensível, empática, por meio da escuta ativa e qualificada que é a base da comunicação terapêutica. As intervenções devem ter foco no enfrentamento eficaz, na resolução de problemas, na esperança e pensamentos positivos a fim de provocar respostas psicoemocionais adaptativas e saudáveis. 


\section{CONSIDERAÇÕES FINAIS}

Neste estudo foram sintetizados alguns principais estudos que retratam a saúde mental dos profissionais de saúde frente a pandemia pelo COVID-19. A saúde mental desses profissionais tem sido apontada como uma grande preocupação devido à frequente exposição ao risco de contaminação, às grandes tomadas de decisões, longas jornadas de trabalho, falta de equipamentos de proteção individual, ampla cobertura da imprensa e baixo estoque de medicamentos. Além disso, a perda de colegas próximos ou familiares também se apresentou como um agravante e pouco se sabe das consequências desse sofrimento psicológico a longo prazo. Baseado nisso é necessário a realização de intervenções psicológicas, a fim de reduzir impactos negativos e promover a saúde mental durante e pós-pandemia, momento este em que as pessoas precisarão se readaptar e lidar com as perdas e transformações emocionais, sociais e econômicas.

\section{REFERÊNCIAS}

1. BANERJEE D. The COVID-19: Crucial role that psychiatrists can play. Ásia J. Psychiatr, 2020; 50(0):1-2.

2. BRASIL. 2020. In: Ministério da Saúde / SAPS - Protocolo de manejo clínico do coronavírus (COVID-19) na atenção primária à saúde. Brasília - DF: Coronavírus COVID-19.

3. BARROSO BIL, et al. Saúde do trabalhador em tempos de COVID-19: reflexões sobre saúde, segurança e terapia ocupacional. João Pessoa: Cadernos Brasileiros de Terapia Ocupacional, Preprint, 2020; 14p.

4. CHAN J. F-W, et al. Um cluster familiar de pneumonia associado ao 2019 novo coronavírus indicando transmissão de pessoa para pessoa: um estudo de um agrupamento familiar. Lancet, 2020; 395(0):514-523.

5. CHEN Q. et al. Mental health care for medical staff in China during the COVID-19 outbreak. Lancet Psychiatry, 2020a; 7(0):15-16.

6. DONG E, DU H, GARDNER L. An interactive web-based dashboard to track COVID-19 in real time. The Lancet. Infectious diseases, v. 3099, n. 20, p. 19-20, 2020.

7. DU J, et al. Psychological symptoms among frontline healthcare workers during COVID-19 outbreak in Wuhan. General Hospital Psychiatry, 2020.

8. EPICENTRO. 2020. SARS-CoV-2: L'epidemiologia per la sanità pubblica. Italia: Istituto Superiore di Sanità.

9. GUIMARÃES AV, BRASIL AM. O adoecimento psíquico e a atividade laboral do profissional de saúde. Projeto de Pesquisa (Trabalho de Conclusãoo de Curso I) - Curso de Graduação em Enfermagem. Centro Universitário de Anápolis, Anápolis, 201;

10. HUANG Y, ZHAO N. Generalized anxiety disorder, depressive symptoms, and sleep quality during COVID-19 outbreak in China: a web-based cross sectional survey. Psychiatry Research, 2020; 288(0):1-6.

11. KANG L, et al. The mental health of medical workers in Wuhan, China dealing with the 2019 novel coronavirus. Lancet Psychiat, 2020; 7(0):7-14.

12. KAVOOR AR, et al. Remote consultations in the era of COVID-19 pandemic: Preliminary experience in a regional Australian public acute mental health care setting. Asian J Psychiatr, 2020; 51(0):2-3.

13. KHALID I, et al. Health workers' emotions, perceived stressors and coping strategies during an MERS-CoV outbreak. Clinical Medicine \& Research, 2016; 14(1):7-14.

14. KHOO EJ. Lessons learned from the COVID-19 pandemic. Acta Pædiatrica, 2020; 0(0):1-3.

15. LAI J, et al. Factors Associated With Mental Health Outcomes Among Health Care Workers Exposed to Coronavirus Disease 2019. JAMANetworkOpen, 2020; 3(3):1-12.

16. LI Q, et al. Early Transmission Dynamics in Wuhan, China, of Novel Coronavirus-Infected Pneumonia. New England Journal of Medicine, p. 1199-1207, 2020.

17. LU W, et al. Psychological status of medical workforce during the COVID-19 pandemic: A cross-sectional study. Psychiatry Research, 2020; 288(0):1-5.

18. MENDES KDS, et al. Revisão integrativa: método de pesquisa para a incorporação de evidências na saúde e na enfermagem. Texto Contexto Enferm, 2008; 17(4):758-764.

19. MINISTÉRIO DA SAÚDE. 2020. In: Centro de Operações de Emergências em Saúde Pública. Infecção Humana pelo Novo Coronavírus (2019-nCoV). Brasília - DF: Boletim Epidemiológico.

20. OPAS. Organização Pan-Americana de Saúde. 2020. In: Folha informativa - COVID-19 (doença causada pelo novo coronavírus).

21. PERDUCANI M, et al. Mental Health Services in Lombardy during COVID-19 outbreak. Psychiatry Research, 2020; 288(0):1-3.

22. PEREIRA MD, et al. The COVID-19 pandemic, social isolation, consequences on mental health and coping strategies: an integrative review. Revista Research, Society and Development, 2020; 9(5):1-29.

23. PHELAN AL, et al. The Novel Coronavirus Originating in Wuhan, China: Challenges for Global Health Governance. JAMANetworkOpen, 2020; 323(8):1-2.

24. REMUZZI A, REMUZZI G. COVID-19 and Italy: what next? The Lancet, 2020; 395(0):11-17.

25. SANTOS CMC, et al. The PICO strategy for the research question construction and evidence search. Revista Latino-Americana de Enfermagem, 2007; 15(3):508-511.

26. SBI. Sociedade Brasileira de Infectologia. 2020. Informe da sociedade brasileira de infectologia (SBI) sobre o novo coronavírus $n^{\circ}$ 10: Perguntas e respostas para profissionais da saúde e para o público em geral. In: São Paulo: Associação Médica Brasileira (AMB).

27. WHO. World Health Organization. 2014. In: Mental health: a state of well-being.

28. ZHANG S, et al. Estimation of the Reproductive Number of Novel Coronavirus (COVID-19) and the Probable Outbreak Size on the Diamond Princess Cruise Ship: A Data-Driven Analysis. International Journal of Infectious Diseases, 2020; 93(0):201-204.

29. ZHU Y, et al. The Risk and Prevention of Novel Coronavirus Pneumonia Infections Among Inpatients in Psychiatric Hospitals. Neurosci Bull, 2020; 36(3):299-302. 